\title{
Echo findings in aortic dissection and car company symbols
}

\author{
Kazuhiro Nishigami
}

Received: 15 June 2009/Revised: 9 July 2009/ Accepted: 13 July 2009/Published online: 18 August 2009

(C) Japanese Society of Echocardiography 2009

Acute aortic dissection is a highly lethal cardiovascular emergency. Diagnostic developments have resulted in earlier recognition and more accurate diagnosis in emergency situations. Especially cardiovascular echo plays an important role in the primary evaluation of patients with suspicious aortic dissections, and the detection of a flap in the aorta can be a key to its diagnosis. Blaivas et al. reported on an ultrasonographic sign of dissection of the ascending aorta in the subxiphoid view that looked like a Mercedes Benz symbol [1]. Although this was the combinational form of a flap and aortic valve, the redissection in patients with aortic dissection can occasionally present three lumens in the aorta, which may also resemble the Mercedes Benz symbol (Fig. 1). The two-dimensional short axis view of the primary dissected aorta with a flap may look like the Mazda symbol (Fig. 2). Consequently, I would like to propose that the Mazda symbol represent the primary dissected aorta with a flap and that the Mercedes Benz symbol represent the redissected aorta with double false lumens. Remembering these car company symbols could facilitate detecting aortic dissections using cardiovascular echo.

\section{Reference}

1. Blaivas M, Sierzenski P. Dissection of the proximal thoracic aorta: a new ultrasonographic sign in the subxiphoid view. Am J Emerg Med. 2002;20:344-8.

K. Nishigami $(\bowtie)$

Division of Cardiology, Saiseikai Kumamoto Hospital

Cardiovascular Center, 5-3-1 Chikami,

Kumamoto 861-4193, Japan

e-mail: kazuhiro-nishigami@saiseikaikumamoto.jp

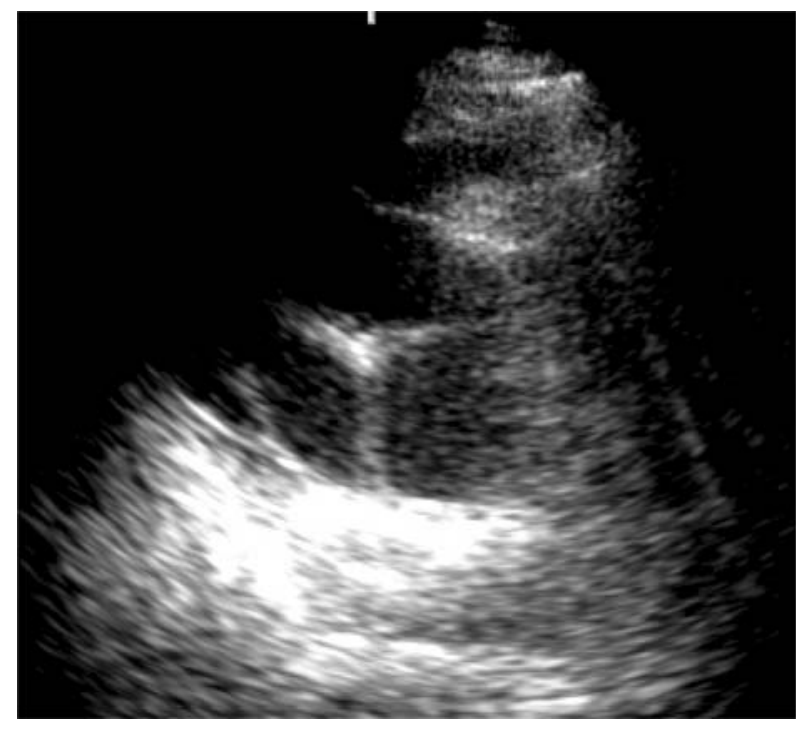

Fig. 1 A left upper parasternal short-axis view of the ascending aorta shows double false lumens, which look like the Mercedes Benz symbol

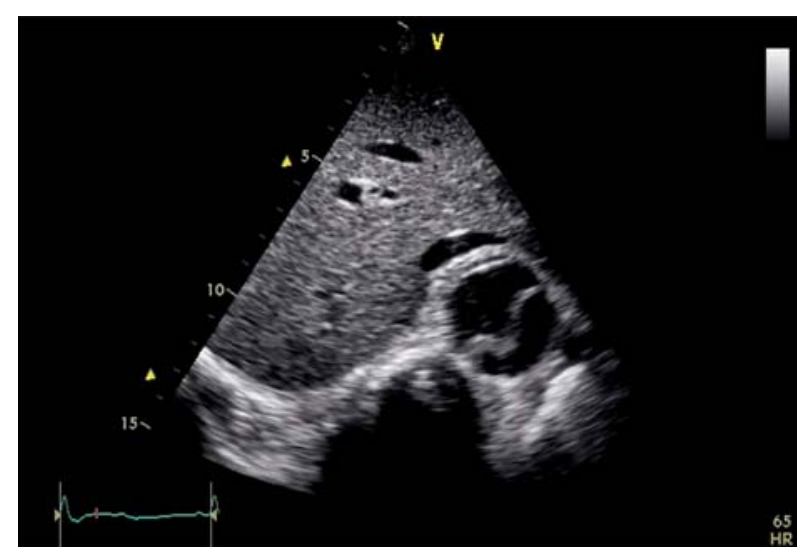

Fig. 2 An upper abdominal short-axis view of the proximal abdominal aorta shows a tortuous flap, which looks like the Mazda symbol 